\title{
COULOMB VERSUS MADELUNG GAP: ORDERING IN A SYSTEM OF POINT CHARGES*
}

\author{
P. Sobkowicz, Z. WILAmowski and J. Kossut \\ Institute of Physics, Polish Academy of Sciences \\ Al. Lotników 32/46, 02-668 Warszawa, Poland
}

\begin{abstract}
Spatial correlations of impurity charges in a mixed valence regime are studied with the use of Monte Carlo simulations. The influence of various kinds of disorder on the one-particle density of states is determined. A continuous transitions from a "soft" Coulomb gap (density of states vanishing only at the chemical potential) to a "hard" gap (with a finite range of energies with vanishing density of states) is found, driven by decreasing amount of built-in disorder in the system. The "hard" Coulomb gap resembles the Madelung gap, found in crystalline arrangements of charges. The similarity reflects the fact that both the Coulomb and Madelung gaps are manifestations of the same phenomenon, resulting from ordering of the positions of point charges, the only difference being the range of correlations.
\end{abstract}

PACS numbers: 71.55.-i, 72.20.Fr

\section{Introduction}

The notion of Coulomb gap (CG) has been introduced in studies of hopping conductivity in classical impurity band (CIB) in lightly doped, compensated semiconductors [1-4]. The term describes a depletion of the single-particle density of states (DOS) near the chemical potential, separating energies of ionized (charged) and occupied (neutral) majority dopant sites. The DOS is derived from electrostatic potential energy values at dopant sitcs. In the case of a neutral site, contributions of all charged sites are taken into account, whereas in the case of a charged site only contributions of other sites are summed. The gap is due to long-range Coulomb interactions in the system of charges of the majority band (donors) and of ionized minority impurities (acceptors). In such systems it is assumed that both donors and acceptors are distributed randomly in space, introducing a "built-in" disorder. In the classical studies of the system, CG has been studied either by means of purely energetical arguments [1], or through Monte Carlo (MC) simulations $[2,4,5]$. In a recent study, Efros postulated that also in an electron liquid without any built-in disorder the same CG exists as in a disordered system [6].

* This work is supported in part by the Committee for Scientific Research under grant no. 204839101 . 
In this work we examine a different semiconductor system where the Coulomb gap is also found, namely a donor resonant with the conduction band in a mixed valence regime [7]. A good example of such a system is offered by iron donors in HgSe. For doping concentrations higher than $4.5 \times 10^{18} \mathrm{~cm}^{-3}$ a part of Fe ions becomes ionized (charged) while the rest remains neutral. Electrons from ionized Fe ions are gathered in the conduction band and provide the charge neutrality.

Due to the electrostatic interactions the impurity charges tend to arrange themselves in an energetically favorable way (by changing distribution of electrons on donor sites). As a result, the charged donors tend to occupy positions as far away from each other as possible. The CG results directly from these spatial rearrangements, and the difference between potential energies at charged and neutral donor sites. Due to the built-in disorder of the spatial distribution of donors in the semiconductor the electrostatic interactions never lead to a perfect arrangement with crystalline order (like in the Wigner crystal) $[8,9]$. It is clear that the degree of correlations in the system depends on the availability of neutral sites. The more sites are present for the charges to choose from, the better the degree of ordering.

\section{Results}

In this work we describe the case when there are many more neutral than charged sites. This may be described by limit of small values of an occupation fraction $f$, defined as the ratio of charged donors to the total number of available donor sites. Our study is based on Monte Carlo simulations, details of which may be found elsewhere $[8,9]$. All presented results were derived for temperature $T=0$.

The results of our investigation may be summarized as follows:

1. For systems with moderate values of the filling factor $f$, e.g., $f=0.3$, the CG has essentially the shape predicted for the CIB (see Fig. 1, line a). The gap has a "soft" character (as predicted by Efros [1]), that is DOS vanishes only at the chemical potential, separating the charged and neutral states.

2. For systems with much smaller $f$ (e.g. $f=0.025$ ) a region of energies without any states (charged or neutral) appears (line $b$ in Fig. 1). This is a result of a much better spatial ordering of charges, made possible by greater availability of sites. The presence of such finite gap in DOS is contrary to the classical predictions of the "soft" nature of the CG. CG for small occupation fractions resembles rather the gap found in DOS of a perfect crystalline arrangement of charges. This gap, which-we call the Madelung gap, is defined similarly to the CG, i.e., it separates the potential values as seen by a lattice site (all sites are equivalent), calculated without contribution of a charge at that site from potential values at all other points in space (which takes into account all the charges in the system). The similarity between the "hard" CG and the Madelung gap is quite understandable when one notes the fact that for small $f$ a quasi-crystalline ordering is clearly visible.

The results presented above show that the shape of the CG depends very strongly on the ability of the system to order itself spatially. The more perfect is the final arrangement of charges, the wider becomes the "hard" CG. Although the 


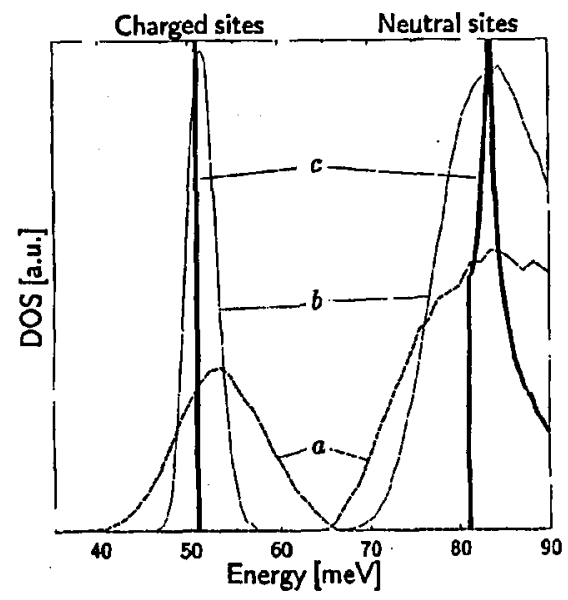

Fig. 1. Examples of DOS calculated for 2D systems with different ratios of the number of charged sites to the total number of sites $(f)$. Line $a: f=0.3$; line $b: f=0.025$. Line $c$ represents DOS for a perfect crystalline arrangement of charges. The high-energy part of DOS, corresponding to neutral sites, has been arbitrarily scaled to fit the figure. The presented results were obtained for $2 \mathrm{D}$ doping density of $1 \times 10^{13} \mathrm{~cm}^{-2}$.

presented figure referred to a two-dimensional system, the same results hold for three-dimensional systems.

In the analysis of our result, it is important to summarize the differences between the physical situation and models used in our case and in studies of CG behavior in the classical impurity band.

In the CIB model, the compensating charge is located on the acceptors, which are assumed to be distributed randomly, and remain always charged. The disorder in the acceptor subsystem, which is not affected by the correlation proccss, influences the ordering phenomena of charges on donors. For example, when there are many more neutral donors than the charged ones, it is energetically advantageous to locate charges on those donors that are close to acceptors, forming $+/$ - dipoles. Pinned by acceptors' positions, these dipoles are distributed randomly in space. We believe that this kind of disorder forbids the formation of a "hard" CG in CIB models, and is responsible for the "soft" nature of CG found in earlier studies $[1,2,4,5]$.

On the other hand, in the resonant donor band model the compensating charge resides in the conduction band. We can assume that the charge is distributed uniformly throughout space. Therefore, it does not introduce any background disorder, so that the degree of ordering in the donor system is influenced only by the temperature fluctuations and availability of neutral sites.

In the limit of continuous distribution of neutral sites (which corresponds to classical electron liquid) at low temperatures a perfect crystalline arrangement of charges is possible and favorable. Our numerical simulations of the classical electron liquid [9] have shown that at low temperatures a "hard" gap in the DOS forms, which is due to the lack of any built-in disorder. 
It should be noted here that our results were obtained for a system of charges interacting through screened, Yukawa-type potential. We found that the formation of the CG is not affected by the screened form of the potential, provided that screening length is of the order of the correlation radius or greater. This stands in contrast with statements that CG is found only in systems with unscreened Coulomb interactions [2].

\section{Conclusions}

The continuous transition from classical, "soft" CG to "hard" gap resembling the Madelung gap for decreasing values of the occupation fraction suggests that the Coulomb and Madelung gap are manifestations of the same phenomenon. At $T=0, \mathrm{CG}$ is simply the Madelung gap closed by the presence of built-in disorder. This disorder may have different origins: presence of disordered charges which do not take part in the ordering process (e.g., charged acceptors) or random distribution of available sites. Depending on the magnitude and nature of the built-in disorder it may be possible or not to obtain a "hard" gap in the DOS. It is important however, to note that the built-in disorder does not close the gap in the same way as thermal fluctuations for $T>0$. In the case of the built-in disorder DOS remains equal to zero at the chemical potential, whereas the thermal fuctuations fill the gap, allowing DOS to become nonzero.

\section{References}

[1] A.L. Efros, B.I. Shklovskii, J. Phys. C, Solid State Phys. 8, L49 (1975).

[2] A.L. Efros, Nguyen Van Lien, B.I. Shklovskii, J. Phys. C: Solid State Phys. 12, 1869 (1979).

[3] A.L. Efros, B.I. Shklovskii, in: Electron-Electron Interactions in Disordered Systems, Eds. A.L. Efros, M. Pollak, Elsevier Sci. Publ., Amsterdam 1985, p. 409.

[4] J.H. Davies, P.A. Lee, T.M. Rice, Phys. Rev. B 29, 4260 (1984).

[5] S.D. Baranovskii, A.L. Efros, B.L. Gelmont, B.I. Shklovskii, J. Phys. C, Solid State Phys. 12, 1023 (1979).

[6] A.L. Efros, Phys. Rev. Lett. 68, 2208 (1992).

[7] Z. Wilamowski, K. Świątek, T. Dietl, J. Kossut, Solid State Commun. 73, 833 (1990).

[8] P. Sobkowicz, Z. Wilamowski, J. Kossut, Semicond. Sci. Technol. 7, 1155 (1992).

[9] P. Sobkowicz, Z. Wilamowski, J. Kossut, to be published in J. Phys., Condens. Matter, 1993. 